\title{
Nanoparticles Get Cool by Light Scattering
}

\author{
Researchers performed 3D cavity cooling of levitated nanoparticles, reaching record low \\ temperatures by utilizing light that scatters off the particles.
}

\section{by Tongcang Li*}

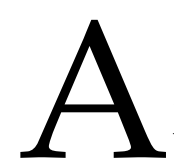

rthur Ashkin pioneered the optical manipulation of small particles with the development of optical tweezers, for which he was awarded the 2018 Nobel Prize in Physics. (See 4 October 2018 Focus story.) The ability to control small particles with tweezers and other optical tools has enabled many breakthroughs in biology, physical chemistry, and atomic, molecular, and optical physics. As part of this trend, researchers have developed ways to "cool" trapped nanoparticles by reducing

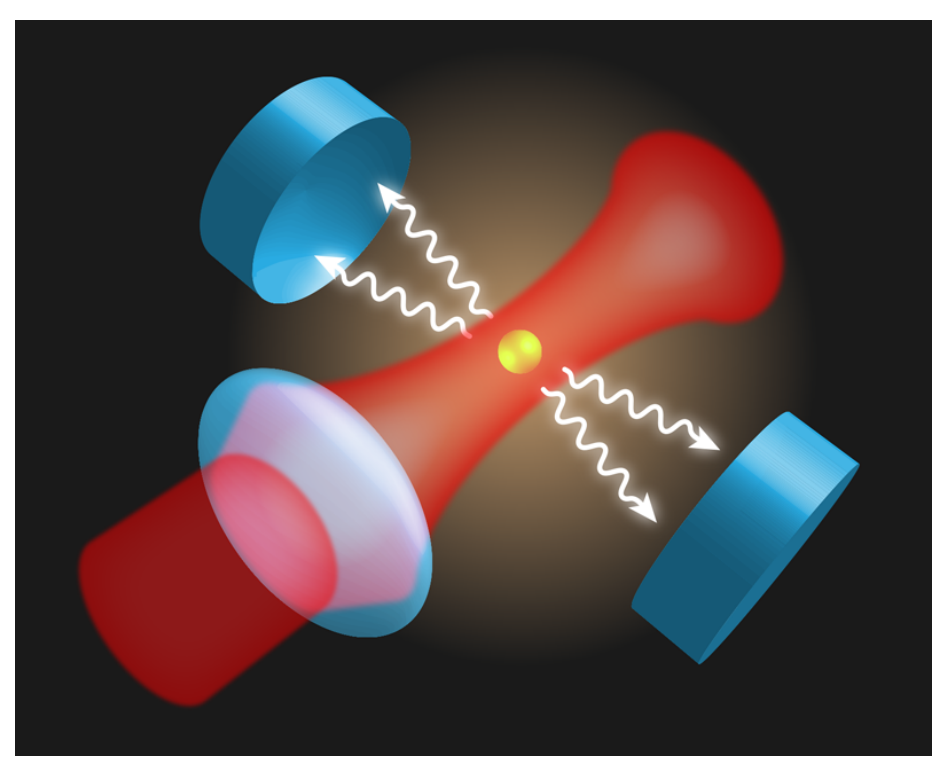

Figure 1: A nanoparticle held by an optical tweezer (red) is placed inside a cavity, depicted by a pair of mirrors (blue). The cavity mode is tuned to a higher frequency than the tweezer light. As such, the photons that scatter off the nanoparticle are preferentially those that "steal" energy from the nanoparticle-effectively cooling it to millikelvin temperatures. (APS/Alan Stonebraker) *Department of Physics and Astronomy and School of Electrical and
Computer Engineering, Purdue University, Indiana, USA the amplitude of their motion within the trap. However, effort is still needed to reach the quantum limit where the motion is dominated by quantum fluctuations. A new method-developed by two independent teams-has taken cooling to the next level by tailoring the light scattering off a nanoparticle held in an optical cavity [1, 2]. Both experiments achieved 3D cavity cooling, while former cavity cooling experiments focused on 1D [3-5]. In one set of experiments, the center-of-mass motion of the nanoparticle was cooled down from room temperature to a minimum effective temperature of a few millikelvin [2], which is much lower than former results achieved by cavity cooling [3-5]. With further developments, this method is promising to reduce the motion of a levitated nanoparticle to its quantummechanical ground state [6].

A levitated microparticle or nanoparticle in vacuum is well isolated from the thermal environment, which is excellent for precision measurements [7]. In recent years, for example, levitated dielectric particles have been used to study nonequilibrium thermodynamics, detect small forces, and search for millicharged particles and other hypothetical phenomena. These systems could also be used to study macroscopic quantum mechanics, but it will require cooling them to near their quantum limit, which is in the microkelvin regime for typical trapping potentials. Two main methods to cool the motion of a levitated dielectric particle are feedback cooling [8] and cavity cooling $[3-5,9,10]$. In feedback cooling, researchers continuously monitor the motion of a dielectric particle with photodetectors, and they use that data to modulate trapping frequencies or apply forces to the nanoparticle that will slow down its motion. Feedback cooling has been able to cool the motion of a nanoparticle to below $1 \mathrm{mK}$ but is currently limited by inefficient motion detection. In cavity cooling, the motion of a nanoparticle affects the frequency of photons in a cavity. Because these photons have a long lifetime in the cavity, they can affect the motion of the nanoparticle at a later time, which provides automatic feedback.

Several experiments have demonstrated cavity cooling of a levitated nanoparticle by driving the cavity with a reddetuned laser, which has a frequency slightly smaller than the resonant frequency of the optical cavity [3-5]. This setup 
favors interactions between photons and the nanoparticle that increase the energy of the photons and thereby decrease the energy of the nanoparticle in $1 \mathrm{D}$ along the cavity axis. In one of the early experiments, optical cavity modes were used for both trapping and cooling of a nanoparticle [3], which turned out to be unstable in vacuum. Later, an ion trap and an optical cavity were combined to achieve stable trapping (by the electric fields of the ion trap) and cooling (by the red-detuned laser) [5]. These experiments were hindered by the weak coupling between the motion of a nanoparticle and the cavity mode. This weak coupling can be offset by driving the cavity mode with strong laser light, but this solution creates a new problem called co-trapping, in which the nanoparticle's position shifts away from the optimal location for cooling.

To achieve efficient 3D cooling, two research teams-one led by Markus Aspelmeyer at the University of Vienna and the other led by René Reimann at the Swiss Federal Institute of Technology (ETH) in Zurich-have used coherent light scattering to realize cavity cooling of the center-of-mass motion of a levitated nanoparticle [1, 2]. In each set of experiments, the researchers used an independent optical tweezer to trap a roughly 140-nm-diameter silica nanoparticle inside an optical cavity (Fig. 1). Because the optical tweezer is tightly focused, its intensity is much higher than the intensity of the cavity mode, which allows stable trapping of the nanoparticle in high vacuum. The teams could control the position of the nanoparticle with an accuracy of a few nanometers along the cavity axis.

For the cooling, the researchers tuned the resonant frequency of the optical cavity to be slightly higher (by about $400 \mathrm{kHz}$ ) than the optical frequency of the trapping laser. This tuning affects the scattering of photons from the nanoparticle. Normally, photons bounce off the nanoparticle like random ping-pong balls, imparting momentum and effectively heating the nanoparticle. However, the cavity prevents this heating by selecting which photons can scatter. Essentially, the photons that scatter are those that bounce off the nanoparticle and steal some of its mechanical vibration energy. This stealing boosts the photon frequency so that it matches the resonant frequency of the cavity. The net effect of this cavity-enhanced coherent scattering should be a reduction in the kinetic energy of the nanoparticle.

Both research teams monitored the motion of the nanoparticle by detecting the slight change of the propagating direction of the trapping laser. The observations showed a slowing in all three dimensions, demonstrating that coherent scattering is an effective 3D cavity cooling method of a levitated nanoparticle. In the ETH Zurich experiment, the motion of a nanoparticle was cooled to a minimum temperature of a few millikelvin at $10^{-5}$ mbar [2]. In the Vienna experiment, the motion of a nanoparticle was cooled to a temperature of about $1 \mathrm{~K}$ at $10^{-2}$ mbar [1].

While both works are important developments in levitated optomechanics, the motions of nanoparticles were still in the classical regime. The cooling results were limited by the heating from surrounding air molecules and the position fluctuation of the optical tweezer [6]. Further reduction of the air pressure and improvement of vibration isolation will enable ground-state cooling. In addition, researchers may use multiple cavity modes to cool the motion more efficiently in 3D [11]. And they may need to develop better methods to accurately measure the effective temperature near the ground state. A levitated nanoparticle in the quantum regime can be used to build a nanoparticle matter-wave interferometer for studying models of the collapse of the wave function, the quantum nature of gravity, and many other applications. We have witnessed rapid progress in levitated optomechanics in the last ten years and expect more fruitful research in this field in the future.

This research is published in Physical Review Letters.

\section{REFERENCES}

[1] U. Delić, M. Reisenbauer, D. Grass, N. Kiesel, V. Vuletić, and M. Aspelmeyer, "Cavity cooling of a levitated nanosphere by coherent scattering," Phys. Rev. Lett. 122, 123602 (2019).

[2] D. Windey, C. Gonzalez-Ballestero, P. Maurer, L. Novotny, O. Romero-Isart, and R. Reimann, "Cavity-based 3D cooling of a levitated nanoparticle via coherent scattering," Phys. Rev. Lett. 122, 123601 (2019).

[3] N. Kiesel, F. Blaser, U. Delic, D. Grass, R. Kaltenbaek, and M. Aspelmeyer, "Cavity cooling of an optically levitated submicron particle," Proc. Natl. Acad. Sci. 110, 14180 (2013).

[4] P. Asenbaum, S. Kuhn, S. Nimmrichter, U. Sezer, and M. Arndt, "Cavity cooling of free silicon nanoparticles in high vacuum," Nat. Commun. 4, 2743 (2013).

[5] J. Millen, P. Z. G. Fonseca, T. Mavrogordatos, T. S. Monteiro, and P.F. Barker, "Cavity cooling a single charged levitated nanosphere," Phys. Rev. Lett. 114, 123602 (2015).

[6] C. Gonzalez-Ballestero, P. Maurer, D. Windey, L. Novotny, R. Reimann, and O. Romero-Isart, "Theory for cavity cooling of levitated nanoparticles via coherent scattering: Master equation approach," arXiv:1902.01282.

[7] Z.-q Yin, A. A. Geraci, and T. Li, "Optomechanics of levitated dielectric particles," Int. J. Mod. Phys. B 27, 1330018 (2013).

[8] T. Li, S. Kheifets, and M. G. Raizen, "Millikelvin cooling of an optically trapped microsphere in vacuum," Nat. Phys. 7, 527 (2011).

[9] O. Romero-Isart, M. L Juan, R. Quidant, and J. I. Cirac, "Toward quantum superposition of living organisms," New J. Phys. 12, 033015 (2010).

[10] D. E. Chang, C. A. Regal, S. B. Papp, D. J. Wilson, J. Ye, O. Painter, H. J. Kimble, and P. Zoller, "Cavity opto-mechanics using an optically levitated nanosphere," Proc. Natl. Acad. Sci. U.S.A. 107, 1005 (2010).

[11] Z.-q. Yin, T. Li, and M. Feng, "Three-dimensional cooling and detection of a nanosphere with a single cavity," Phys. Rev. A 83, 013816 (2011).

10.1103/Physics. 12.34 